\title{
Special Weingarten surfaces foliated by circles*
}

\author{
Rafael López \\ Departmento de Geometra y Topologa \\ Universidad de Granada \\ 18071 Granada (Spain) \\ e-mail:rcamino@ugr.es
}

MSC 2000 subject classification: 53A05, 53C40

\begin{abstract}
In this paper we study surfaces in Euclidean 3-space foliated by pieces of circles and that satisfy a Weingarten condition of type $a H+b K=c$, where $a, b$ and $c$ are constant and $H$ and $K$ denote the mean curvature and the Gauss curvature respectively. We prove that a such surface must be a surface of revolution, a Riemann minimal surface or a generalized cone.
\end{abstract}

\section{Introduction}

A surface $S$ in 3-dimensional Euclidean space $\mathbb{R}^{3}$ is called a Weingarten surface if there is some relation between its two principal curvatures $\kappa_{1}$ and $\kappa_{2}$, that is, if there is a smooth function $W$ of two variable such that $W\left(\kappa_{1}, \kappa_{2}\right)=0$. In particular, if $K$ and $H$ denote respectively the Gauss and the mean curvature of $S, W\left(\kappa_{1}, \kappa_{2}\right)=0$ implies a relation $U(K, H)=0$. In this work we study Weingarten surfaces that satisfy the simplest case for $U$, that is, that $U$ is of linear type:

$$
a H+b K=c,
$$

where $a, b$ and $c$ are constant with $a^{2}+b^{2} \neq 0$. We say then that $S$ is a special Weingarten surface and we abbreviate it by SW-surface. Constant mean curvature surfaces $(b=$ $0)$ or constant Gauss curvature surfaces $(a=0)$ are SW-surfaces. The classification of Weingarten surfaces is almost completely open today. Weingarten introduced this kind of surfaces in the context of the problem of finding all surfaces isometric to a given surface of

${ }^{*}$ Partially supported by MEC-FEDER grant no. MTM2004-00109. 
revolution $[21,22]$. Along the history, they have been of interest for geometers $[2,6,8,9,20]$ and more recently in $[7,12,15,17,19]$. Applications of Weingarten surfaces on computer aided design and shape investigation can seen in [1].

Among all surfaces, a first class is the one of surfaces of revolution. In such case, Equation (1) leads to an ordinary differential equation and its study is then simplified to find the profile curve (for example, see [17] if $c=0$ ). Recall that in the case of constancy of mean curvature or Gauss curvature, they are well known [3,4]. A more general family of surfaces of revolution are the cyclic surfaces.

Definition 1.1 A cyclic surface in Euclidean space $\mathbb{R}^{3}$ is a surface determined by a smooth uniparametric family of pieces of circles.

Thus, a cyclic surface is a surface foliated by pieces of circles meaning that there is a one-parameter family of planes which meet $S$ in these pieces of circles. The planes are not assumed parallel, and if two circles should lie in planes that happen to be parallel, the circles are not assumed coaxial. We point out that a sphere is a surface such that any family of planes (parallel or not) intersects it in circles. The study of cyclic hypersurfaces with constant curvature in different ambient spaces was re-opened recently by Jagy [10, 11]. See also [14].

The aim of this paper is if, besides the surfaces of revolution, there exist new cyclic SWsurfaces. Our work is motivated by it happens in the cases of constant mean curvature or constant Gauss curvature and that can summarized as follows. In both settings, the surface is an open of a sphere or the planes of the foliation are parallel $[5,13,16]$. When the planes of the foliation are parallel, then either it is a subset of a surface of revolution or it is a subset of one of the following non-rotational surfaces:

1. It is one of the examples of minimal surfaces discovered by Riemann [18]. This happens when $H=0$.

2. It is a generalized cone, that is, a cyclic surface where the circle centres lie in a straight-line and the radius function is linear. In this case, $K=0$. Locally, it can be parametrized by $\mathbf{X}(u, v)=(f(u), g(u), u)+r(u)(\cos (v), \sin (v), 0)$, where $f, g$ and $r>0$ are linear functions on $u[13]$.

The first result that we shall obtain here states that in a cyclic SW-surface, the foliation planes must be parallel (except the trivial case of a sphere).

Theorem 1.2 If $S$ is a $S W$-surface foliated by pieces of circles lying in a one-parameter family of planes, then either $S$ is a subset of a round sphere or the planes in the family are parallel. 
Once proved this result, we continue the search of cyclic SW-surfaces in the situation of parallel planes. The conclusion that we shall obtain is that the circles of the foliation must be coaxial, unless the known cases of constant mean curvature or constant Gauss curvature.

Theorem 1.3 Let $S$ be a $S W$-surface foliated by pieces of circles lying in a one-parameter family of parallel planes. Then either $S$ is a piece of a surface of revolution or $S$ is part of one of the Riemann minimal examples or a generalized cone.

As conclusion of Theorems 1.2 and 1.3, we have

Corollary 1.4 A surface in Euclidean space foliated by pieces of circles and that satisfies $a$ condition of type $a H+b K=c$, where $a, b$ and $b$ are constant must be a surface of revolution, a Riemann minimal surface or a generalized cone.

Therefore, although the family of surfaces satisfying the equation $a H+b K=c$ is larger than the one of constant mean curvature and constant Gauss curvature and so, one could think the existence of cyclic non-rotational surfaces for each three real numbers $(a, b, c)$, Corollary 1.4 says that this only occurs for the known cases of $H=0$ or $K=0$. In this sense, we can view these two class of surfaces as a special set of surfaces in the family of SW-surfaces.

Corollary 1.5 Riemann examples of minimal surfaces and generalized cones are the only non-rotational cyclic surfaces that satisfy a Weingarten relation of type $a H+b K=c$.

This paper is organized as follows. In Section 2, we recall some concepts of the classical differential geometry of surfaces in $\mathbb{R}^{3}$. Section 3 and 4 are devoted to show Theorems 1.2 and 1.3 respectively.

\section{Preliminaries}

In this section, we fix some notation on local classical differential geometry on surfaces. Let $S$ be a surface in $\mathbb{R}^{3}$ and consider $\mathbf{X}=\mathbf{X}(u, v)$ a local parametrization of $S$ defined in the $(u, v)$-domain. Let $N$ denote the unit normal vector field on $S$ given by

$$
N=\frac{\mathbf{X}_{u} \wedge \mathbf{X}_{v}}{\left|\mathbf{X}_{u} \wedge \mathbf{X}_{v}\right|}, \quad \mathbf{X}_{u}=\frac{\partial \mathbf{X}}{\partial u}, \mathbf{X}_{v}=\frac{\partial \mathbf{X}}{\partial v}
$$


where $\wedge$ stands the cross product of $\mathbb{R}^{3}$. In each tangent plane, the induced metric $\langle$,$\rangle is$ determined by the first fundamental form

$$
I=\langle d \mathbf{X}, d \mathbf{X}\rangle=E d u^{2}+2 F d u d v+G d v^{2},
$$

with differentiable coefficients

$$
E=\left\langle\mathbf{X}_{u}, \mathbf{X}_{u}\right\rangle, \quad F=\left\langle\mathbf{X}_{u}, \mathbf{X}_{v}\right\rangle, \quad G=\left\langle\mathbf{X}_{v}, \mathbf{X}_{v}\right\rangle .
$$

The shape operator of the immersion is represented by the second fundamental form

$$
I I=-\langle d N, d \mathbf{X}\rangle=e d u^{2}+2 f d u d v+g d v^{2},
$$

with

$$
e=\left\langle N, \mathbf{X}_{u u}\right\rangle, \quad f=\left\langle N, \mathbf{X}_{u v}\right\rangle, \quad g=\left\langle N, \mathbf{X}_{v v}\right\rangle .
$$

Under this parametrization $\mathbf{X}$, the mean curvature $H$ and the Gauss curvature $K$ have the classical expressions

$$
H=\frac{e G-2 f f+g E}{2\left(E G-F^{2}\right)}, \quad K=\frac{e g-f^{2}}{E G-F^{2}} .
$$

Let us denote by $[,$,$] the determinant in \mathbb{R}^{3}$ and put $W=E G-F^{2}$. Then $H$ and $K$ write as

$$
\begin{gathered}
H=\frac{G\left[\mathbf{X}_{u}, \mathbf{X}_{v}, \mathbf{X}_{u u}\right]-2 F\left[\mathbf{X}_{u}, \mathbf{X}_{v}, \mathbf{X}_{u v}\right]+E\left[\mathbf{X}_{u}, \mathbf{X}_{v}, \mathbf{X}_{v v}\right]}{2 W^{3 / 2}}:=\frac{H_{1}}{2 W^{3 / 2}}, \\
K=\frac{\left[\mathbf{X}_{u}, \mathbf{X}_{v}, \mathbf{X}_{u u}\right]\left[\mathbf{X}_{u}, \mathbf{X}_{v}, \mathbf{X}_{v v}\right]-\left[\mathbf{X}_{u}, \mathbf{X}_{v}, \mathbf{X}_{u v}\right]^{2}}{W^{2}}:=\frac{K_{1}}{W^{2}}
\end{gathered}
$$

Using (2) and (3), a SW-surface satisfies the condition

$$
a \frac{H_{1}}{2 W^{3 / 2}}+b \frac{K_{1}}{W^{2}}=c
$$

or, equivalently,

$$
a H_{1} W^{1 / 2}=2\left(c W^{2}-b K_{1}\right)
$$

Squaring both sides, we have

$$
a^{2} H_{1}^{2} W-4\left(c W^{2}-b K_{1}\right)^{2}=0 .
$$

The proof of Theorems 1.2 and 1.3 involves explicit computations of identity (4) and subsequent manipulations. As we shall see in the next two sections, Equation (4) reduces to an expression that can be written as a linear combination of the functions $\cos (j v)$ and $\sin (j v), 0 \leq j \leq 8$, whose coefficients $A_{j}$ and $B_{j}$ are functions on the $u$-variable. Therefore, they must vanish in some $u$-interval. The work then is to compute explicitly these coefficients by successive manipulations. The author was able to obtain the results using the symbolic program Mathematica to check his work. The computer was used in each calculation several times, giving understandable expressions of the coefficients $A_{j}$ and $B_{j}$. 


\section{Proof of Theorem 1.2}

In this section, we follow the same ideas as in [16] and [10] for the case that the mean curvature is constant. For this, we wish to construct an appropriate coordinate system to our foliation of the surface $S$. Let us denote by $\Pi_{u}$ these planes in such way $S \cap \Pi_{u}$ is each piece of the circles of the foliation. Consider a smooth unit vector field $Z$ that is normal to the planes $\Pi_{u}$. Next, we take a particular integral curve $\Gamma=\Gamma(u)$ of $Z$ parametrized by arclength, that is, $\mathbf{t}(u):=\Gamma^{\prime}(u)=Z(\Gamma(u))$, where $\mathbf{t}$ is the unit tangent vector to $\Gamma$. Consider the Frenet frame of the curve $\Gamma,\{\mathbf{t}, \mathbf{n}, \mathbf{b}\}$, where $\mathbf{n}$ and $\mathbf{b}$ denote the normal and binormal vectors respectively.

Locally we parametrize $S$ by

$$
\mathbf{X}(u, v)=\mathbf{c}(u)+r(u)(\cos v \mathbf{n}(u)+\sin v \mathbf{b}(u)),
$$

where $r=r(u)>0$ and $\mathbf{c}=\mathbf{c}(u)$ denote respectively the radius and centre of each $u$-circle of the foliation. Consider the Frenet equations of the curve $\Gamma$ :

$$
\begin{array}{rlc}
\mathbf{t}^{\prime} & = & \kappa \mathbf{n} \\
\mathbf{n}^{\prime} & = & -\kappa \mathbf{t}+\sigma \mathbf{b} \\
\mathbf{b}^{\prime} & = & -\sigma \mathbf{n}
\end{array}
$$

where the prime ' denotes the derivative with respect to the $u$-parameter and $\kappa$ and $\sigma$ are the curvature and torsion of $\Gamma$, respectively.

Also, set

$$
\mathbf{c}^{\prime}=\alpha \mathbf{t}+\beta \mathbf{n}+\gamma \mathbf{b}
$$

where $\alpha, \beta, \gamma$ are smooth functions on $u$.

By using the Frenet equations and (6), a straightforward computation of (4) shows that it can be expressed by a trigonometric polynomial on $\cos (j v), \sin (j v)$. Exactly, there exist smooth functions on $u$, namely $A_{j}$ and $B_{j}$, such that Equation (4) writes as

$$
A_{0}+\sum_{j=1}^{8}\left(A_{j}(u) \cos (j v)+B_{j}(u) \sin (j v)\right)=0, \quad u \in I, v \in J
$$

Since this is an expression on the independent trigonometric terms $\cos (j v)$ and $\sin (j v)$, all coefficients $A_{j}, B_{j}$ must be zero.

In the reasoning to prove Theorem 1.2, we shall assume that the planes of the foliation are not parallel and then, our objective will be to show that the surface is included in a sphere of Euclidean space. Thus, in our assumption, the integral curve $\Gamma$ is not a straight line perpendicular to each $u$-plane and so, the curvature $\kappa$ is not vanishing. The surface 
$S$ is part of a sphere if and only if each point of $S$ is the same distance from a fixed point $c_{0}$ in $\mathbb{R}^{3}$. We shall recognize it in a few number of situations as the following: (i) the centre curve $\mathbf{c}$ is constant as well as the radius function $r(u)$ or; (ii) the curve $\mathbf{c}$ can written as a combination of $\mathbf{t}, \mathbf{n}$ and $\mathbf{b}$ in such way that the parametrization (5) is now $\mathbf{X}(u, v)=c_{0}+\varphi(u, v) \mathbf{t}(u)+\phi(u, v) \mathbf{n}(u)+\psi(u, v) \mathbf{b}(u)$ where $\left|\mathbf{X}(u, v)-c_{0}\right|=\sqrt{\varphi^{2}+\phi^{2}+\psi^{2}}$ is a non-zero constant, the radius of the sphere that we are looking for.

In the proof, we distinguish two situations according the value $c$ in (1).

\subsection{Case $c=0$ in the relation $a H+b K=c$.}

Without loss of generality we assume that $a=1$. The coefficients $A_{8}$ and $B_{8}$ are

$$
\begin{aligned}
A_{8} & =\frac{1}{32} \kappa^{2} r^{8}\left(\beta^{6}-\left(15 \gamma^{2}+\kappa^{2}\left(b^{2}-3 r^{2}\right)\right) \beta^{4}\right. \\
& -\left(15 \gamma^{4}+6 \gamma^{2} \kappa^{2}\left(b^{2}-3 r^{2}\right)+\kappa^{4} r^{2}\left(-2 b^{2}+3 r^{2}\right)\right) \beta^{2} \\
+ & \left(\gamma^{2}-\kappa^{2} r^{2}\right)\left(\gamma^{2}+\kappa^{2}\left(b^{2}-r^{2}\right)\right) . \\
B_{8} & =\frac{1}{16} \beta \gamma \kappa^{2} r^{8}\left(3 \beta^{4}-2 \beta^{2}\left(5 \gamma^{2}+\kappa^{2}\left(b^{2}-3 r^{2}\right)\right)\right. \\
& \left.+\left(\gamma^{2}-\kappa^{2} r^{2}\right)\left(3 \gamma^{2}+\kappa^{2}\left(2 b^{2}-3 r^{2}\right)\right)\right),
\end{aligned}
$$

From $B_{8}=0$, we discuss three cases.

1. Case $\beta=0$ in some sub-interval of $I$. Then

$$
A_{8}=-\frac{1}{32} \kappa^{2} r^{8}\left(\gamma^{2}-\kappa^{2} r^{2}\right)^{2}\left(\gamma^{2}+\kappa^{2}\left(b^{2}-r^{2}\right)\right) .
$$

If $\gamma^{2}=\kappa^{2} r^{2}$, then

$$
A_{6}=-\frac{9}{8} b^{2} \kappa^{6} r^{10}\left(\alpha^{2}-r^{2}\right), \quad B_{6}= \pm \frac{9}{4} b^{2} \alpha \kappa^{6} r^{10} r^{\prime}
$$

which it is implies that $\alpha=0$ and $r$ is a constant function. But then $A_{4}=-2 r^{12} b^{2} \kappa^{8}$, obtaining a contradiction. As conclusion, and from $A_{8}=0$, we have that $\gamma=$ $\pm \kappa \sqrt{r^{2}-b^{2}}$. Now

$$
A_{7}=-\frac{1}{16} b^{4} \alpha \kappa^{7} r^{9}, \quad B_{7}= \pm \frac{b^{4} \kappa^{7} r^{10} r^{\prime}}{16 \sqrt{r^{2}-b^{2}}}
$$

Thus $r$ is a constant function and $\alpha=0$. Then $A_{5}= \pm r^{9} \kappa^{7} b^{4} \tau \sqrt{r^{2}-b^{2}} / 4$, that is, $\tau=0$ or $r= \pm b$. If $\tau=0, A_{4}=r^{8} b^{2}\left(r^{2}-b^{2}\right)\left(5 r^{2}+3 b^{2}\right) \kappa^{8} / 8$, which it leads to 
$r= \pm b$, and so, $\gamma=0$. Then (6) implies that the curve of centres $\mathbf{c}(u)$ is constant, $\mathbf{c}(u)=c_{0}$ for some $c_{0} \in \mathbb{R}^{3}$. From (5), $\left|\mathbf{X}(u, v)-c_{0}\right|=b^{2}$, that is, our surface is an open of a sphere of radius $|b|$.

We summarize this case by saying that if the foliation planes are not parallel, then the surface is a piece of a sphere.

2. Case $\gamma=0$ in some sub-interval of $I$. The coefficient $A_{8}$ is

$$
A_{8}=\frac{1}{32} \kappa^{2} r^{8}\left(\beta^{2}+\kappa^{2} r^{2}\right)^{2}\left(\beta^{2}+\kappa^{2}\left(r^{2}-b^{2}\right)\right) .
$$

Then $\beta^{2}=\kappa^{2}\left(b^{2}-r^{2}\right)$. Without loss of generality, we assume that $\beta=\kappa \sqrt{b^{2}-r^{2}}$. It follows that

$$
A_{7}=-\frac{1}{16} b^{4} \kappa^{7} r^{9}\left(\alpha+\frac{r r^{\prime}}{\sqrt{b^{2}-r^{2}}}\right) .
$$

Then $\alpha=-r r^{\prime} / \sqrt{b^{2}-r^{2}}$. From (6), we can write

$$
\mathbf{c}^{\prime}=-\frac{r r^{\prime}}{\sqrt{b^{2}-r^{2}}} \mathbf{t}+\kappa \sqrt{b^{2}-r^{2}} \mathbf{n}=\left(\sqrt{b^{2}-r^{2}} \mathbf{t}\right)^{\prime} .
$$

Then there exists $c_{0} \in \mathbb{R}^{3}$ such that $\mathbf{c}=c_{0}+\sqrt{b^{2}-r^{2}} \mathbf{t}$ and the parametrization $\mathbf{X}$ of the surface is now

$$
\mathbf{X}(u, v)=c_{0}+\sqrt{b^{2}-r^{2}} \mathbf{t}+r(\cos (v) \mathbf{n}+\sin (v) \mathbf{b}) .
$$

This implies that $\left|\mathbf{X}(u, v)-c_{0}\right|=b^{2}$ and $S$ is again a piece of a sphere of radius $|b|$. In this setting, the same conclusion is obtained as in the above case.

3. Case $\beta \gamma \neq 0$. From $B_{8}=0$ in (9), we can calculate $\beta^{2}$ :

$$
\beta^{2}=\frac{1}{3}\left(5 \gamma^{2}+b^{2} \kappa^{2}-3 \kappa^{2} r^{2} \pm A\right)
$$

where $A=\sqrt{16 \gamma^{4}+4 b^{2} \gamma^{2} \kappa^{2}+b^{4} \kappa^{4}-12 \gamma^{2} \kappa^{2} r^{2}}$. We consider the sign ' + ' in the value of $\beta^{2}$ (similarly with the choice -). Let us put it into $A_{8}$ and taking into account that $\kappa \neq 0$, we obtain the following identity:

$416 \gamma^{6}+b^{6} \kappa^{6}+96 \gamma^{4} \kappa^{2}\left(b^{2}-3 r^{2}\right)+18 \gamma^{2} \kappa^{4} b^{4}=-\left(b^{4} \gamma^{4}+112 \gamma^{4}+16 \gamma^{2} \kappa^{2}\left(b^{2}-3 r^{2}\right)\right) A$.

Squaring both sides and after some manipulations, we obtain

$$
\left(\gamma^{2}-\kappa^{2} r^{2}\right)\left(\left(4 \gamma^{2}+b^{2} \kappa^{2}\right)^{2}-16 \gamma^{2} \kappa^{2} r^{2}\right)=0 .
$$

We discuss each one of the possibilities: 
(a) $\gamma^{2}=\kappa^{2} r^{2}$. Using (10), $\beta^{2}=2 \kappa^{2}\left(b^{2}+2 r^{2}\right) / 3$ and returning with the computation of $A_{8}$ in (8), we have

$$
A_{8}=-\frac{1}{216} \kappa^{8} r^{8}\left(b^{2}+2 r^{2}\right)\left(b^{2}+8 r^{2}\right)^{2} .
$$

Then $A_{8}=0$ yields a contradiction.

(b) $\left(4 \gamma^{2}+b^{2} \kappa^{2}\right)^{2}-16 \gamma^{2} \kappa^{2} r^{2}=0$. From here, we obtain the value of $\gamma^{2}$ :

$$
\gamma^{2}=\frac{\kappa^{2}}{4}\left(r \pm \sqrt{r^{2}-b^{2}}\right)^{2}
$$

Then the value of $\beta^{2}$ in $(10)$ is

$$
\beta^{2}=\frac{1}{12} \kappa^{2}\left(-5 b^{2}+2 r^{2}+14 r \sqrt{r^{2}-b^{2}}\right) .
$$

From (8), Equation $A_{8}=0$ gives

$$
\left(r^{2}-b^{2}\right)\left(b^{4}-14 b^{2} r^{2}+16 r^{4}+\left(16 r^{3}-6 r b^{2}\right) \sqrt{r^{2}-b^{2}}\right)=0,
$$

in particular, $r$ is a constant function. The manipulation with the second factor implies that it cannot vanish. Thus $r^{2}=b^{2}$. But then $\beta^{2}=-\kappa^{2} b^{2} / 4$ : contradiction.

\subsection{Case $c \neq 0$ in the relation $a H+b K=c$.}

Without loss of generality, we shall assume that $c=1$. The computation of the coefficients $A_{8}$ and $B_{8}$ gives

$$
A_{8}=-\frac{1}{32} r^{8} x_{1}, \quad B_{8}=\frac{1}{16} \beta \gamma r^{8} x_{2},
$$

where

$$
\begin{aligned}
x_{1} & =\beta^{8}-\left(28 \gamma^{2}+\kappa^{2}\left(a^{2}+2 b-4 r^{2}\right)\right) \beta^{6} \\
& +\left(70 \gamma^{4}+15 \gamma^{2} \kappa^{2}\left(a^{2}+2 b-4 r^{2}\right)+\kappa^{4}\left(b^{3}-3\left(a^{2}+2 b\right) r^{2}+6 r^{4}\right)\right) \beta^{4} \\
& +\left(-28 \gamma^{6}-15 \gamma^{4} \kappa^{2}\left(a^{2}+2 b-4 r^{2}\right)+\kappa^{6} r^{2}\left(2 b^{2}-3\left(a^{2}+2 b\right) r^{2}+4 r^{4}\right)\right. \\
& \left.-6 \gamma^{2} \kappa^{4}\left(b^{2}-3\left(a^{2}+2 b\right) r^{2}+6 r^{4}\right)\right) \beta^{2} \\
& +\left(\gamma^{2}-\kappa^{2} r^{2}\right)^{2}\left(\gamma^{4}+\gamma^{2} \kappa^{2}\left(a^{2}+2 b-2 r^{2}\right)+\kappa^{4}\left(b^{2}-\left(a^{2}+2 b\right) r^{2}+r^{4}\right)\right) . \\
x_{2}= & -4 \beta^{6}+\left(28 \gamma^{2}+3 \kappa^{2}\left(a^{2}+2 b-4 r^{2}\right) \beta^{4}\right. \\
- & 2\left(14 \gamma^{4}+5 \gamma^{2} \kappa^{2}\left(a^{2}+2 b-4 r^{2}\right)+\kappa^{4}\left(b^{2}-3\left(a^{2}+2 b\right) r^{2}+6 r^{4}\right) \beta^{2}\right. \\
+ & \left(\gamma^{2}-\kappa^{2} r^{2}\right)\left(4 \gamma^{4}+\gamma^{2} \kappa^{2}\left(3 a^{2}+6 b-8 r^{2}\right)+\kappa^{4}\left(2 b^{2}-3\left(a^{2}+2 b\right) r^{2}+4 r^{4}\right) .\right.
\end{aligned}
$$


We discard the cases $a=0$ or $b=0$, corresponding to the known situations of (non-zero) constant mean or Gauss curvature: in such case, $S$ is a piece of a sphere. From $B_{8}=0$, we discuss the following cases:

1. Case $\gamma=0$. From $A_{8}=0$,

$$
\left.\beta^{4}-\kappa^{2}\left(a^{2}+2 b-2 r^{2}\right) \beta^{2}+\kappa^{4}\left(b^{2}-\left(a^{2}+2 b\right) r^{2}+r^{4}\right)\right)=0 .
$$

Then

$$
\beta^{2}=\frac{1}{2} \kappa^{2}\left(a^{2}+2 b-2 r^{2} \pm a \sqrt{a^{2}+4 b}\right) .
$$

In particular, $a^{2}+4 b \geq 0$. In the reasoning, we shall suppose the positive sign in \pm of the expression of $\beta^{2}$. According to the value of $a^{2}+4 b$, we distinguish two cases.

(a) $a^{2}+4 b=0$. From the value of $\beta^{2}$, the coefficient $B_{5}$ gives

$$
B_{5}=\frac{1}{128} a^{4} \kappa^{5} r^{7} \sqrt{a^{2}-4 r^{2}}\left(\alpha \sqrt{a^{2}-4 r^{2}}+2 r r^{\prime}\right)^{2}=0 .
$$

If $\sqrt{a^{2}-4 r^{2}}=0, \beta=0$ and we are in the case " $\beta=0 "$ that it will be studied in the second case of this subsection. Thus,

$$
\alpha=-\frac{2 r r^{\prime}}{\sqrt{a^{2}-4 r^{2}}}
$$

and this allows us to write

$$
\mathbf{c}^{\prime}=\left(\frac{\sqrt{a^{2}-4 r^{2}}}{2} \mathbf{t}\right)^{\prime}
$$

As a consequence, there exists some fixed vector $c_{0}$ such that

$$
\mathbf{c}=c_{0}+\frac{\sqrt{a^{2}-4 r^{2}}}{2} \mathbf{t} .
$$

Then the parametrization $\mathbf{X}$ in (5) gives $\left|\mathbf{X}(u, v)-c_{0}\right|^{2}=a^{2} / 4$, that is, the surface is a open of a sphere of radius $|a| / 2$.

In the second case, the computation of the coefficient $B_{5}=0$ implies

$$
\alpha \sqrt{a^{2}-4 r^{2}}+2 r r^{\prime}=0 \quad \text { or } \quad \tau=0 .
$$

obtaining the same result as above. 
(b) $a^{2}+4 b>0$. The coefficient $A_{7}$ is

$$
B_{7}=\frac{1}{64} a A B \kappa^{5} r^{9}\left(\alpha \kappa^{2}-\kappa \beta^{\prime}+\kappa^{\prime} \beta\right)=0,
$$

with

$$
\left.A=2 b+a\left(a+\sqrt{a^{2}+4 b}\right) \quad B=a^{3}+4 a b+\left(a^{2}+2 b\right) \sqrt{a^{2}+4 b}\right) .
$$

The number $A$ does not vanish and $B=0$ only if $a^{2}+4 b=0$. As conclusion,

$$
\alpha=\left(\frac{\beta}{\kappa}\right)^{\prime} .
$$

Following (6), the derivative of the curve $\mathbf{c}$ is

$$
\mathbf{c}^{\prime}=\left(\frac{\beta}{\kappa}\right)^{\prime} \mathbf{t}+\beta \mathbf{n}=\left(\frac{\beta}{\kappa} \mathbf{t}\right)^{\prime} .
$$

From (5), the parametrization of the surface is

$$
\mathbf{X}(u, v)=c_{0}+\frac{\beta}{\kappa} \mathbf{t}+r(\cos (v) \mathbf{n}+\sin (v) \mathbf{b}),
$$

for some fixed vector $c_{0}$. Using the value of $\beta^{2}$, we have,

$$
\left|\mathbf{X}(u, v)-c_{0}\right|^{2}=\frac{\beta^{2}}{\kappa^{2}}+r^{2}=\frac{1}{2} a^{2}+b+\frac{a}{2} \sqrt{a^{2}+4 b} .
$$

This means that the surface is an open of a certain sphere.

2. Case $\beta=0$. Now

$$
A_{8}=-\frac{1}{32} r^{8}\left(\gamma^{2}-\kappa^{2} r^{2}\right)^{2} x_{1}, \quad A_{7}=-\frac{1}{16} \alpha \kappa r^{9}\left(\gamma^{2}-\kappa^{2} r^{2}\right) y_{1},
$$

where

$$
\begin{gathered}
x_{1}=\gamma^{4}+\kappa^{2} \gamma^{2}\left(a^{2}+2 b-2 r^{2}\right)+\kappa^{4}\left(b^{2}-\left(a^{2}+2 b\right) r^{2}+r^{4}\right) . \\
y_{1}=8 \gamma^{4}+\left(7\left(a^{2}+2 b\right)-16 r^{2}\right) \kappa^{2} \gamma^{2}+\kappa^{4}\left(6 b^{2}-7\left(a^{2}+2 b\right) r^{2}+8 r^{4}\right) .
\end{gathered}
$$

We discuss three possibilities:

(a) Case $\gamma^{2}=\kappa^{2} r^{2}$. Then

$$
A_{6}= \pm \frac{9}{8} b^{2} \kappa^{6} r^{10}\left(\alpha^{2}-r^{2}\right), \quad B_{6}= \pm \frac{9}{8} b^{2} \alpha \kappa^{6} r^{10} r^{\prime}
$$

Then $\alpha=0$ and $r$ is a constant function. Then $A_{4}=-2 r^{12} b^{2} \kappa^{8}$, giving a contradiction. 
(b) Case $x_{1}=\alpha=0$. We know that

$$
\gamma^{2}=\frac{1}{2} k^{2}\left( \pm a \sqrt{a^{2}+4 b}-\left(a^{2}+2 b-2 r^{2}\right)\right) .
$$

Without loss of generality, we assume the sign + in \pm . Then

$$
B_{7}=\frac{1}{32} a r^{9} C \kappa^{3}\left(\gamma^{2}-\kappa^{2} r^{2}\right)\left(\kappa \gamma^{\prime}-\kappa^{\prime} \gamma\right),
$$

where $C=a^{3}+4 a b-\left(a^{2}+2 b\right) \sqrt{a^{2}+4 b}$. For each pair $(a, b)$ of real numbers, $C \neq 0$ except when $a^{2}+4 b=0$. Then $\kappa \gamma^{\prime}-\kappa^{\prime} \gamma=0$. From this equation, we conclude that $r$ is a constant function. We discuss both situations according to the value of $a^{2}+4 b$ :

i. Let $a^{2}+4 b=0$. Equation $A_{4}=0$ implies $16 r^{4}+8 a^{2} r^{2}-3 a^{4}=0$, that is, $4 r^{2}-a^{2}=0$. Thus $\gamma=0$ and this case was studied above.

ii. Let $a^{2}+4 b>0$. Now $A_{5}=0$ implies $\tau=0$. After some manipulations, $A_{4}=0$ and $A_{2}=0$ give $\kappa=0$ : contradiction.

(c) Case $x_{1}=y_{1}=0$. Then $8 x_{1}-y_{1}=0$ means

$$
\left(a^{2}+2 b\right) \gamma^{2}+\kappa^{2}\left(2 b^{2}-\left(a^{2}+2 b\right) r^{2}\right)=0 \text {. }
$$

In particular, $a^{2}+2 b \neq 0$ and $\gamma^{2}=\kappa^{2}\left(\left(a^{2}+2 b\right) r^{2}-2 b^{2}\right) /\left(a^{2}+2 b\right)$. With this value of $\gamma^{2}$,

$$
x_{1}=-\frac{a^{2} b^{2}\left(a^{2}+4 b\right) \kappa^{4}}{\left(a^{2}+2 b\right)^{2}}=0,
$$

which it is a contradiction.

3. Case $\beta \gamma \neq 0$. This case is more difficult in the computations due to that the expressions are very cumbersome. We only give the details. It follows from the expressions of $A_{8}$ and $B_{8}$ in (8) that $x_{1}=x_{2}=0$ : see (12) and (13). We begin to compute the value of $\beta^{2}$. For this, we define $x_{3}:=4 x_{1}+\beta^{2} x_{2}=0$ and $x_{4}:=$ $4 x_{3}-\left(84 \gamma^{2}+\kappa^{2}\left(a^{2}+2 b-4 r^{2}\right)\right) x_{2}$. Now $x_{4}$ is a 2-degree polynomial on $\beta^{2}$ and we can calculate $\beta^{2}$ :

$$
\beta^{2}=\frac{\xi \pm \sqrt{\xi^{2}-\eta \lambda \zeta}}{\eta}
$$

where

$$
\begin{aligned}
\xi & =960 \gamma^{6}+320 \gamma^{4} \kappa^{2}\left(a^{2}+2 b-4 r^{2}\right)+\kappa^{6}\left(b^{2}\left(a^{2}+2 b\right)-\left(3 a^{4}+12 a^{2} b+4 b^{2}\right) r^{2}\right) \\
& +5 \gamma^{2} \kappa^{4}\left(a^{4}+4 a^{2} b+12 b^{2}-32 r^{2}\left(a^{2}+2 b-2 r^{2}\right)\right), \\
\eta & =1344 \gamma^{4}+\left(3 a^{4}+12 a^{2} b+4 a^{2}\right) \kappa^{4}+80 \gamma^{2} \kappa^{2}\left(a^{2}+2 b-4 r^{2}\right), \\
\lambda & =\gamma^{2}-\kappa^{2} r^{2} \\
\zeta & =320 \gamma^{6}+80 \gamma^{4} \kappa^{2}\left(3 a^{2}+6 b-6 r^{2}\right)+\kappa^{6}\left(2 b^{2}\left(a^{2}+2 b\right)-\left(3 a^{4}+12 a^{2} b+4 b^{2}\right) r^{2}\right) \\
& +\gamma^{2} \kappa^{4}\left(3 a^{4}+12 a^{2} b+164 b^{2}-240\left(a^{2}+2 b\right) r^{2}+320 r^{4}\right) .
\end{aligned}
$$


For each one of the two values of $\beta^{2}$, we return to $x_{2}=0$ obtaining the following:

$$
\lambda \mu \eta^{3} \rho=0,
$$

where

$$
\begin{aligned}
\mu= & 16 \gamma^{4}+a^{2}\left(a^{2}+4 b\right) \kappa^{4}+8 \gamma^{2} \kappa^{2}\left(a^{2}+2 b-2 r^{2}\right) \\
\rho= & \left(16 \gamma^{4}+32 \kappa r \gamma^{3}+4 \kappa^{2}\left(a^{2}+2 b+4 r^{2}\right) \gamma^{2}+4\left(a^{2}+2 b\right) \kappa^{3} r \gamma+b^{2} \kappa^{4}\right) \\
& \left(16 \gamma^{4}-32 \kappa r \gamma^{3}+4 \kappa^{2}\left(a^{2}+2 b+4 r^{2}\right) \gamma^{2}-4\left(a^{2}+2 b\right) \kappa^{3} r \gamma+b^{2} \kappa^{4}\right) .
\end{aligned}
$$

From Equation (15), we have four cases to discuss in such way that we can compute the value of $\gamma^{2}$ and, next, putting it in (14), the value of $\beta^{2}$. For instance, if $\lambda=0$, that is, $\gamma^{2}-\kappa^{2} r^{2}=0$, the value of $\beta^{2}$ is

$$
\beta^{2}=2 \kappa^{2} \frac{2 r^{2}\left(a^{4}+2 a^{2} b+28 b^{2}+80\left(a^{2}+2 b\right) r^{2}\right)+b^{2}\left(a^{2}+2 b\right)}{3 a^{4}+12 a^{2}+b+4 b^{2}+80\left(a^{2}+2 b\right) r^{2}+1024 r^{4}} .
$$

On the other hand, $x_{2}$ writes now as

$$
x_{2}=\beta^{2}\left(-4 \beta^{4}+\beta^{2} \kappa^{2}\left(3 a^{2}+6 b+16 r^{2}\right)-2 \kappa^{4}\left(b^{2}+2\left(a^{2}+2 b\right) r^{2}\right)\right)
$$

and it follows that

$$
\beta^{2}=\frac{\kappa^{2}}{8}\left(3 a^{2}+6 b+16 r^{2} \pm \sqrt{9 a^{4}+36 a^{2} b+4 b^{2}+32 a^{2} r^{2}+64 b r^{2}+256 r^{4}}\right) .
$$

Comparing both values of $\beta^{2}$, we know then $r^{2}$ is one of the following values:

$$
r^{2}=-\frac{a^{2}\left(a^{2}+4 b\right)}{8\left(a^{2}+2 b\right)}, \quad r^{2}=\frac{-1}{16}\left(a^{2}+2 b \pm \sqrt{a^{2}\left(a^{2}+4 b\right)}\right) .
$$

If we analyze, for example, the first value of $r^{2}$, we know that

$$
\beta^{2}=\frac{1}{2}\left(a^{2}+2 b\right) \kappa^{2} \quad \gamma^{2}=-\frac{a^{2}\left(a^{2}+4 b\right)}{8\left(a^{2}+2 b\right)} \kappa^{2} .
$$

Now $A_{7}=0$ implies $\alpha \kappa^{7}=0$, that is, $\alpha=0$. Equations $A_{5}=0$ and $B_{5}=0$ give $\tau \kappa^{7}=0$, and so, $\tau=0$. With $j=4$,

$$
\begin{gathered}
A_{4}=\left(21 a^{6}+130 a^{4} b+240 a^{2} b^{2}+96 b^{3}\right) \kappa^{8}=0, \\
B_{4}=\left(21 a^{4}+88 a^{2} b+96 b^{2}\right) \kappa^{8}=0,
\end{gathered}
$$

which it would imply $\kappa=0$, obtaining the desired contradiction.

Remark 3.1 Throughout our reasoning in the case $c \neq 0$, it has appeared, as a particular case, that $a^{2}+4 b>0$ (or $a^{2}+4 b c>0$ in the Weingarten relation (1)). This is not casual: Weingarten surfaces that satisfy this property were treated by the very Hopf in [9] by their special properties (see also [7]). 


\section{Proof of Theorem 1.3}

Once we have proved Theorem 1.2, we consider SW-surfaces foliated by pieces of circles in parallel planes. The conclusion that we shall arrive is that either (i) the circles of the foliation must coaxial, that is, the surface is an open subset of a surface of revolution or (ii) it is part of a Riemann minimal surface $(H=0)$ or of a generalized cone $(K=0)$. This is the statement of Theorem 1.3, which it will be proved in this section. Because our reasoning is of local character, we can assume the planes are parallel to the $x_{1} x_{2}$-plane. Therefore, the surface $S$ writes as

$$
\mathbf{X}(u, v)=(f(u), g(u), u)+r(u)(\cos v, \sin v, 0)
$$

where $f, g$ and $r$ are smooth functions in some $u$-interval $I$ and $r>0$ denotes the radius of each circle of the foliation. With this parametrization, $S$ is a surface of revolution if and only if $f \mathrm{y} g$ are constant functions. If we compute (4), we obtain

$$
\sum_{j=0}^{8} A_{j}(u) \cos (j v)+B_{j}(u) \sin (j v)=0 .
$$

Again, the functions $A_{j}$ and $B_{j}$ on $u$ must vanish on $I$.

In our reasoning, we shall assume that the foliated circles are not coaxial and that that $b^{2}+c^{2} \neq 0$ and $a^{2}+c^{2} \neq 0$ (which it would yield that $S$ is part of a Riemann example or of a generalized cone). With these assumptions, we will arrive to a contradiction. As in the above section, we distinguish two cases according to the value of $c$ in the relation $a H+b K=c$.

\subsection{Case $c=0$.}

In this particular situation, the sum in (16) is until $j=4$.

1. First, we consider the cases that one of the functions $f$ or $g$ is constant. For simplicity, we shall consider $f^{\prime}=0$ in some interval. Then $A_{4}$ writes as

$$
A_{4}=\frac{1}{8} a^{2} r^{6} g^{\prime 2}\left(r g^{\prime \prime}-2 r^{\prime} g^{\prime}\right)
$$

As $g^{\prime} \neq 0$, we have that $r g^{\prime \prime}-2 r^{\prime} g^{\prime}=0$. Then $g^{\prime}=\lambda r^{2}$ for some positive constant $\lambda \neq 0$. Now

$$
A_{2}=-\frac{1}{2} \lambda^{2} r^{8}\left(a^{2} r^{2} A^{2}-16 b^{2} r^{2}\right), \quad B_{1}=2 \lambda r^{7} r^{\prime}\left(a^{2} r A^{2}-8 b^{2} r^{\prime \prime}\right),
$$


where

$$
A=1+\lambda^{2} r^{4}+r^{\prime 2}-r r^{\prime \prime} .
$$

From Equation $A_{2}=0$ and the value of $A$, we discard the case that $r$ is a constant function. Thus, the combination of $A_{2}=0$ and $B_{1}=0$ leads to that the function $r$ satisfies the ordinary differential equation $r r^{\prime \prime}-2 r^{\prime 2}=0$. Then

$$
r(u)=\frac{\alpha}{u+\beta}, \quad \alpha, \beta \in \mathbb{R} .
$$

But then $A_{2}=0$ gives a polynomial on $u$ given by

$$
16 b^{2}(u+\beta)^{6}-a^{2}\left((u+\beta)^{4}-\alpha^{2}+\lambda^{2} \alpha^{4}\right)^{2}=0, \quad \forall u \in I
$$

and whose leading coefficient, corresponding to $u^{8}$, is $-a^{2}$ : this is a contradiction. This means that the assumption that $f$ is constant is impossible.

2. We assume that both $f$ and $g$ are not constant functions. Then $f^{\prime}, g^{\prime} \neq 0$. The computation of $B_{4}$ gives now

$$
B_{4}=\frac{1}{4} a^{2} r^{6}\left(r g^{\prime} f^{\prime \prime}+f^{\prime}\left(-4 g^{\prime} r^{\prime}+r g^{\prime \prime}\right)\right)\left(-2 f^{\prime 2} r^{\prime}+r f^{\prime} f^{\prime \prime}+g^{\prime}\left(2 g^{\prime} r^{\prime}-r g^{\prime \prime}\right)\right) .
$$

We have two possibilities.

(a) Case $r g^{\prime} f^{\prime \prime}+f^{\prime}\left(-4 g^{\prime} r^{\prime}+r g^{\prime \prime}\right)=0$. Then

$$
f^{\prime \prime}=\frac{f^{\prime}\left(4 g^{\prime} r^{\prime}-r g^{\prime \prime}\right)}{r g^{\prime}},
$$

and the coefficient $A_{4}=0$ gives

$$
A_{4}=\frac{a^{2} r^{6}\left(f^{\prime 2}+g^{\prime 2}\right)^{2}}{8 g^{\prime 2}}\left(r g^{\prime \prime}-2 g^{\prime} r^{\prime}\right)^{2} .
$$

Thus $r g^{\prime \prime}-2 g^{\prime} r^{\prime}=0$, that is, $g^{\prime}=\lambda r^{2}$ with $\lambda>0$. Using (17), the same occurs for $f^{\prime}: f^{\prime}=\mu r^{2}, \mu>0$. The computation of $A_{2}$ and $A_{1}$ leads to

$$
\begin{gathered}
A_{2}=-\frac{1}{2}\left(\lambda^{2}-\mu^{2}\right) r^{8}\left(a^{2} r^{2} A^{2}-16 b^{2} r^{2}\right), \\
A_{1}=2 \mu r^{7} r^{\prime}\left(-8 b^{2} r^{\prime \prime}+a^{2} r A^{2}\right),
\end{gathered}
$$

where the value of $A$ is now

$$
A=1+\left(\lambda^{2}+\mu^{2}\right) r^{4}+r^{\prime 2}-r r^{\prime \prime} .
$$

From the expression of $A$ together $A_{2}=0$, we conclude that $r$ is not a constant function. By combining $A_{2}=0$ and $A_{1}=0$, we obtain $r r^{\prime \prime}-2 r^{\prime 2}=0$ again. The contradiction is obtained as in the case that $f$ is a constant function. 
(b) Case $-2 f^{\prime 2} r^{\prime}+r f^{\prime} f^{\prime \prime}+g^{\prime}\left(2 g^{\prime} r^{\prime}-r g^{\prime \prime}\right)=0$. From here, we obtain $f^{\prime \prime}$ and putting it into $A_{4}$, it gives

$$
A_{4}=-\frac{a^{2} r^{6}\left(f^{\prime 2}+g^{\prime 2}\right)^{2}}{8 f^{\prime 2}}\left(r g^{\prime \prime}-2 g^{\prime} r^{\prime}\right)^{2}
$$

Then $r g^{\prime \prime}-2 g^{\prime} r^{\prime}=0$ : we are now in the position of the above case.

\subsection{Case $c \neq 0$.}

The computation of $A_{8}$ and $B_{8}$ give respectively:

$$
\begin{gathered}
A_{8}=-\frac{1}{32} c^{2} r^{8}\left(f^{\prime 8}-28 f^{\prime 6} g^{\prime 2}+70 f^{\prime 4} g^{\prime 4}-28 f^{\prime 2} g^{\prime 6}+g^{\prime 8}\right) . \\
B_{8}=\frac{1}{4} c^{2} r^{8} f^{\prime} g^{\prime}\left(-f^{\prime 6}+7 f^{\prime 4} g^{\prime 2}-7 f^{\prime 2} g^{\prime 4}+g^{\prime 6}\right) .
\end{gathered}
$$

Since $\alpha(u)=(f(u), g(u))$ is not a constant planar curve, we reparametrize it by the arclength, that is, $(f(u), g(u))=(x(\phi(u), y(\phi(u))$, where

$$
f^{\prime}(u)=\phi^{\prime}(u) \cos (\phi(u)), \quad g^{\prime}(u)=\phi^{\prime}(u) \sin (\phi(u)), \quad \phi^{\prime 2}=f^{\prime 2}+g^{\prime 2} .
$$

With this change of variable, the functions $A_{8}$ and $B_{8}$ write now as:

$$
\begin{aligned}
& A_{8}=-\frac{1}{32} c^{2} r^{8} \phi^{\prime 8} \cos (8 \phi(u)) . \\
& B_{8}=-\frac{1}{32} c^{2} r^{8} \phi^{\prime 8} \sin (8 \phi(u)) .
\end{aligned}
$$

As $c \neq 0$ and $r>0$, we conclude that $\phi^{\prime}=0$ on some interval, that is, $\alpha$ is a constant curve, obtaining a contradiction.

This finishes the proof of Theorem 1.3.

We end with a comment when $c=0$. For the cases of constant mean curvature or constant Gauss curvature, the same above computations give:

1. Constant mean curvature $(b=0)$.

$$
f^{\prime \prime}=\lambda r^{2}, \quad g^{\prime \prime}=\mu r^{2} \quad 1+\left(\lambda^{2}+\mu^{2}\right) r^{4}+r^{2}-r r^{\prime \prime}=0,
$$

which it gives the Riemann examples of minimal surfaces $\left(\lambda^{2}+\mu^{2} \neq 0\right)$ and the catenoid $(\lambda=\mu=0)$.

2. Constant Gauss curvature $(a=0)$.

$$
f^{\prime \prime}=g^{\prime \prime}=r^{\prime \prime}=0,
$$

that is, the functions $f, g$ and $r$ are linear on $u$ and so, the surface is a generalized cone. 


\section{References}

[1] B. van-Brunt, K. Grant, Potential applications of Weingarten surfaces in CAGD. I: Weingarten surfaces and surface shape investigation, Comput. Aided Geom. Des. 13 (1996), 569-582.

[2] S. S. Chern, Some new characterizations of the Euclidean sphere, Duke Math. J. 12, (1945) 279-290.

[3] C. Delaunay, Sur la surface de révolution dont la courbure moyenne est constante, J. Math. Pure Appl. 6 (1841), 309-320.

[4] L. Eisenhart, Differential Geometry, Gin and Co. Boston, 1909.

[5] A. Enneper, Die cyklischen Flächen, Z. Math. Phys. 14 (1869), 393-421.

[6] P. Hartman, W. Winter, Umbilical points and W-surfaces, Am. J. Math. 76 (1954), 502-508.

[7] J.A. Gálvez, A. Martínez, F. Milán, Linear Weingarten Surfaces in $\mathbb{R}^{3}$, Monatsh. Math. 138 (2003), 133-144.

[8] H. Hopf, Über Flächen mit einer Relation zwischen den Hauptkrümmungen, Math. Nachr. 4 (1951), 232-249.

[9] H. Hopf, Differential Geometry in the Large, Lecture Notes in Math, vol. 1000, 1983, Berlin, Springer-Verlag.

[10] W.C. Jagy, Minimal hypersurfaces foliated by spheres, Michigan Math. J. 38 (1991), 255-270.

[11] W.C. Jagy, Sphere-foliated constant mean curvature submanifolds, Rocky Mount. J. Math. 28 (1998), 983-1015.

[12] W. Kühnel, M. Steller, On closed Weingarten surfaces, Monatsh. Math. 146 (2005), 113-126.

[13] R. López, Cyclic surfaces of constant Gauss curvature, Houston Math. J. 27 (2001), 799-805.

[14] R. López, Cyclic hypersurfaces of constant curvature, Advanced Studies in Pure Mathematics 34, Minimal Surfaces, Geometric Analysis and Symplectic Geometry (2002),185-199.

[15] R. López, On linear Weingarten surfaces, preprint 2006.

[16] J.C.C. Nitsche, Cyclic surfaces of constant mean curvature, Nachr. Akad. Wiss. Gottingen Math. Phys. II, 1 (1989), 1-5.

[17] B. Papantoniou, Classification of the surfaces of revolution whose principal curvatures are connected by the relation $A \kappa_{1}+B \kappa_{2}=0$ where $A$ or $B$ is diferent from zero, Bull. Calcutta Math. Soc. 76 (1984), 49-56.

[18] B. Riemann, Über die Flächen vom Kleinsten Inhalt be gegebener Begrenzung. Abh. Königl. Ges. Wissensch. Göttingen Mathema. Cl. 13 (1868), 329-333.

[19] H. Rosenberg, R. Sa Earp, The geometry of properly embedded special surfaces in $\mathbb{R}^{3}$; e.g., surfaces satisfying $a H+b K=1$, where $a$ and $b$ are positive, Duke Math. J. 73 (1994), 291-306.

[20] K. Voss, Über geschlossene Weingartensche Flächen, Math. Annalen. 138 (1959), 42-54. 
[21] J. Weingarten, Ueber eine Klasse auf einander abwickelbarer Fläachen, J. Reine Angew. Math. 59 (1861), 382-393.

[22] J. Weingarten, Ueber die Flächen, derer Normalen eine gegebene Fläche berühren, J. Reine Angew. Math. 62 (1863), 61-63. 